\title{
MINIMAL (MAX,+) REALIZATION OF CONVEX SEQUENCES*
}

\author{
STÉPHANE GAUBERT ${ }^{\dagger}$, PETER BUTKOVIČ $\ddagger$ AND RAYMOND CUNINGHAME-GREEN $\ddagger$
}

Abstract. We show that the minimal dimension of a linear realization over the $(\max ,+)$ semiring of a convex sequence is equal to the minimal size of a decomposition of the sequence as a supremum of discrete affine maps. The minimal-dimensional realization of any convex realizable sequence can thus be found in linear time. The result is based on a bound in terms of minors of the Hankel matrix.

Key words. max plus algebra, minimal realization, rational sequences, discrete-event systems

AMS subject classifications. 93B20, 15A15

PII. S036301299528534X

1. Introduction. A classical problem consists in studying infinite sequences $h_{0}, h_{1}, \ldots$ with values in a semiring $(\mathcal{S}, \oplus, \otimes)$, generated by a finite device. The simplest and most studied class is probably that of realizable or recognizable sequences, obtained as the scalar output of finite-dimensional recurrent $\mathcal{S}$-linear systems:

$$
x_{0}=b, \quad x_{k+1}=A x_{k}, \quad h_{k}=c x_{k}, \quad k=0,1, \ldots,
$$

where $A \in \mathcal{S}^{n \times n}, b, x_{0}, x_{1}, \ldots \in \mathcal{S}^{n \times 1}, c \in \mathcal{S}^{1 \times n}$ for some positive integer $n$, and where concatenation denotes the matrix product as usual. ${ }^{1}$ Equivalently,

$$
h_{k}=c A^{k} b, \quad k=0,1, \ldots
$$

The triple $(A, b, c)$ is called a linear realization or representation of the sequence $h$, and $n$ is called the dimension of the realization $(A, b, c)$. By the Kleene-Schützenberger theorem [3], realizable sequences coincide with rational sequences (sequences of coefficients of rational series). The theory of these sequences is much developed in the case of fields (particularly $\mathcal{S}=\mathbb{R}$ or $\mathbb{C}$ ). The case of realizable sequences over the semiring of nonnegative reals $\left(\mathbb{R}^{+},+, \times\right)$is also much studied in connection with probability measures and Markov chains [13, 22]. Here, we are concerned with realizable sequences over the " $(\max ,+)$ " semiring $\mathbb{R}_{\max } \stackrel{\text { def }}{=}(\mathbb{R} \cup\{-\infty\}, \oplus, \otimes)$, with max as addition $(a \oplus b \stackrel{\text { def }}{=} \max (a, b))$ and + as product $(a \otimes b \stackrel{\text { def }}{=} a+b)$. The interest in realizable sequences over $\mathbb{R}_{\max }$ arises from at least two fields.

a) In discrete-event systems theory, it is known that an important subclass of man-made systems with synchronization features (manufacturing systems, transportations networks, etc.) can be modeled by input-output variants of the dynamics (1.1), namely,

$$
x_{k+1}=A x_{k} \oplus b u_{k+1}, \quad y_{k}=c x_{k}, \quad k \in \mathbb{Z},
$$

where $u_{k}, y_{k} \in \mathbb{R} \cup\{-\infty\}$. In the case of manufacturing systems, typically, the input $u_{k}$ represents the availability date of the $k$ th unit of raw material, and $y_{k}$ represents

\footnotetext{
${ }^{*}$ Received by the editors April 28, 1995; accepted for publication (in revised form) October 17, 1996.

http://www.siam.org/journals/sicon/36-1/28534.html

${ }^{\dagger}$ INRIA, Domaine de Voluceau, BP 105, 78153 Le Chesnay cédex, France (Stephane. Gaubert@inria.fr).

${ }^{\ddagger}$ School of Mathematics and Statistics, University of Birmingham, Birmingham B15 2TT, UK (p.butkovic@bham.ac.uk, r.a.cuninghame-green@bham.ac.uk).

${ }^{1}$ E.g., $\left(A x_{k}\right)_{i}=\oplus_{j=1}^{n} A_{i j} \otimes\left(x_{k}\right)_{j}, c x_{k}=\oplus_{j=1}^{n} c_{j} \otimes\left(x_{k}\right)_{j}$, etc.
} 
the availability date of the $k$ th finished part, while the vector $x_{k}$ represents the dates of completion of internal events. It is not difficult to see that the minimal output $y$ of (1.3) corresponding to the earliest behavior of the system is given by the supconvolution

$$
y_{k}=\bigoplus_{p \in \mathbb{N}} h_{p} \otimes u_{k-p}=\sup _{p \in \mathbb{N}}\left[h_{p}+u_{k-p}\right],
$$

so that the realizable sequence $h$ determines the input-output relation of (1.3). As in the case of conventional linear systems, the sequence $h_{0}, h_{1}, \ldots$ is called the impulse response of the system, for it coincides with the output $y$ associated with the impulse input $u: u_{k}=0$ if $k=0, u_{k}=-\infty$ otherwise. See [1] for a complete presentation.

b) In dynamic programming, the simplest stationary deterministic Markovian decision problem with finite state space $Q=\{1, \ldots, n\}$, transition reward $A: Q \times Q \rightarrow$ $\mathbb{R} \cup\{-\infty\}$, initial reward $c: Q \rightarrow \mathbb{R} \cup\{-\infty\}$, final reward $b: Q \rightarrow \mathbb{R} \cup\{-\infty\}$, and horizon $k$, writes

$$
\max _{q_{0}, \ldots, q_{k}}\left[c\left(q_{0}\right)+\sum_{i=1}^{k} A\left(q_{i-1}, q_{i}\right)+b\left(q_{k}\right)\right] .
$$

Identifying $A, b, c$ with matrices of appropriate sizes, it is immediately seen that the optimal reward in horizon $k$, given by (1.5), coincides with $h_{k}=c A^{k} b$.

In this paper, we are concerned with the minimal realization problem, which, given a sequence $h$, consists in finding a (linear) realization $(A, b, c)$ with minimal dimension. For instance, in the Markov decision context, the minimal realization problem asks whether or not there exists another decision problem $\left(A^{\prime}, b^{\prime}, c^{\prime}\right)$ of type (1.5), with state space $Q^{\prime}$ of strictly smaller cardinality but the same reward history $h_{0}, h_{1}, \ldots$ as $(A, b, c)$. In the context of discrete-event systems, this is a natural problem, which consists in finding a minimal internal realization of the system (1.4), known only by its input-output relation $u \rightarrow y$. This has often interesting practical interpretations: loosely speaking, the nonminimality of the triple $(A, b, c)$ arises from the existence of nontrivial temporal relations between the different physical events in the system. Particularly, nonminimalities occur when some component of the system (a particular machine or process) which plays a physical role in the production process is invisible from the performance evaluation point of view, i.e., when the normal functioning of this process will never delay the output dates due to the existence of margins. This striking phenomenon is illustrated on the cover page of the book [1], to which the reader is referred for more motivation.

Unlike in the field's case, the minimal realization problem over $\mathbb{R}_{\max }$ is not solved by rank arguments. It is indeed very much analogous to that of the nonnegative realization (over the usual algebra) [14] for which only partial solutions are known. We refer the reader to Olsder [29, 28], Cuninghame-Green [6], Qi and Chen [31], Gaubert [15, Chap. VI], De Schutter and De Moor [10, 11, 12, 9] for existing results (realization procedures, bounds, heuristics, reduction of the partial realization problem to an extended linear complementarity problem). See also [1, sections 1.3 and 9.2.3]. In the present paper, we characterize the minimal dimension of a realization for the subclass of convex realizable sequences, extending a result given by Cuninghame-Green and Butkovič [7] for the strictly convex case. The proof requires the minor bound given by Gaubert in [15, Chapter VI; 16], together with a classical majorization result [26].

It is worth noting that the convexity assumption, although restrictive, is natural with respect to a subclass of discrete-event systems. Input-output systems (1.4) with 
affine realizable impulse response, $h_{k}=\alpha+\beta \times k$, can be interpreted as (delayed) flow limiters [1, section 6.2.2], i.e., as periodic systems, with minimal interevent delay $\beta$ and transfer delay $\alpha$. When building complex discrete-event systems from simple ones, one uses in particular the synchronization (or parallel composition) operation [1], which corresponds to the pointwise max of the impulse responses. Since a convex map is the upper envelope of its tangent lines, it is not difficult to see that realizable convex responses correspond exactly to parallel composition of finitely many delayed flow limiters.

Finally, we refer the reader interested in an overview of the theory and applications of the $(\max ,+)$ semiring to $[5,19,1,27,21]$. General results on semirings can be found in [18].

2. Statement of the result. A sequence $h_{0}, h_{1}, \ldots \in \mathbb{R}$ is convex if

$$
k \geq 1 \Rightarrow h_{k+1}-h_{k} \geq h_{k}-h_{k-1} .
$$

It follows from the well-known periodicity property of $(\max ,+)$ realizable sequences (see [5], [1, Theorem 3.112], [17, Theorem 7]) that a realizable convex sequence is eventually periodic of period one; that is, there exists $N \in \mathbb{N}$ and $\lambda \in \mathbb{R}$ such that

$$
k \geq N \Rightarrow \quad h_{k+1}=\lambda+h_{k} .
$$

In what follows, $N$ will always stand for the least natural number satisfying (2.1) and will be called the length of the transient of the sequence. A max-polynomial [8] is the function

$$
p(x)=\max _{1 \leq i \leq r}\left(\alpha_{i}+\beta_{i} x\right),
$$

with $\alpha_{i} \in \mathbb{R}, \beta_{i} \in \mathbb{R}$. The name "polynomial" refers to the notation of the semiring $\mathbb{R}_{\max }: a \oplus b=\max (a, b), a \otimes b=a+b$, and, for $n \in \mathbb{N}, a^{n}=\underbrace{a \otimes \cdots \otimes a}_{n \text { times }}(=n \times a)$.

Then, (2.2) becomes

$$
p(x)=\bigoplus_{i=1}^{r} \alpha_{i} \otimes x^{\beta_{i}} .
$$

Note that we extend the exponent notation and use $x^{\beta_{i}}$ for $x \times \beta_{i}$, even when $\beta_{i} \notin \mathbb{N}$ : unlike in the conventional case, maxpolynomials may have real (nonintegral) exponents. We say that $p$ is a polynomial realization of $h$ if for all nonnegative integers $k$, $p(k)=h_{k}$. We denote by $\operatorname{mpr}(h)$ the minimal number of monomials of a polynomial realization of $h$ (i.e., the minimal value of $r$ ). By convention, $\operatorname{mpr}(h)=+\infty$ when $h$ does not admit a polynomial realization. Denote by $\mathrm{mlr}(h)$ the minimal dimension of a linear realization (with $\operatorname{mlr}(h)=+\infty$ if $h$ is not realizable). The main result of this paper is the following characterization which solves the minimal realization problem for convex sequences.

THEOREM 2.1. For every convex sequence $h$ there holds

$$
\operatorname{mpr}(h)=\operatorname{mir}(h) .
$$

Note that the theorem states in particular that the existence of a polynomial realization is equivalent to that of a linear one, for convex sequences.

Efficient computation of $\operatorname{mpr}(h)$ is not difficult: given a convex sequence $h$ with the length of the transient $N$, the algorithm given in the Appendix provides a minimal polynomial realization in time $O(N)$. 
The inequality $\operatorname{mlr}(h) \leq \operatorname{mpr}(h)$ is immediate: if $p$ is a polynomial realization of $h$ of type (2.3), then $h_{k}=c \operatorname{diag}(\beta)^{k} b$, where $b$ denotes the $r \times 1$ matrix with entries $0, c$ the $1 \times r$ matrix with entries $\alpha_{1}, \ldots, \alpha_{r}$, and $\operatorname{diag}(\beta)$ the $r \times r$ matrix with diagonal entries $\beta_{1}, \ldots, \beta_{r}$ and off-diagonal elements equal to $-\infty$. The proof of the reverse inequality will use a bound in terms of determinants and Hankel matrices, which we introduce next.

3. Minor bound for the minimal dimension of realization. Recall that the Hankel matrix $[13,3]$ associated with the sequence $h_{0}, h_{1}, \ldots$ is the $\mathbb{N} \times \mathbb{N}$-matrix

$$
\mathcal{H}=\left(\mathcal{H}_{i j}\right), \mathcal{H}_{i j}=h_{i+j} \text { for all } i, j=0,1, \ldots
$$

A classical result for the minimal realization problem over fields states that the minimal dimension of any realization of a sequence $h$ is equal to the rank of its Hankel matrix, which can be defined equivalently as the cardinality of a basis of the vector space generated by the rows (or columns) of $\mathcal{H}$ or as the maximal size of a square submatrix of $\mathcal{H}$ with nonzero determinant.

Over a general (commutative) semiring $\mathcal{S}$, several nonequivalent rank notions exist, ${ }^{2}$ which do not characterize the minimal dimension of realization but only provide bounds. Here, we will need the rank notion originating from determinants and bideterminants over semirings.

Given a positive integer $n$, let $\mathfrak{S}_{n}^{+}, \mathfrak{S}_{n}^{-}$, respectively, denote the sets of even and odd permutations on $\{1, \ldots, n\}$ (we use the concepts of even and odd permutations in the conventional sense [4]). The positive and negative determinants of an $n \times n$ matrix $A$ with entries from a (commutative) semiring $\mathcal{S}$ are defined as follows:

$$
\begin{aligned}
& \operatorname{det}^{+} A=\bigoplus_{\sigma \in \mathfrak{S}_{n}^{+}} \bigotimes_{i=1}^{n} A_{i \sigma(i)}, \\
& \operatorname{det}^{-} A=\bigoplus_{\sigma \in \mathfrak{S}_{n}^{-}} \bigotimes_{i=1}^{n} A_{i \sigma(i)} .
\end{aligned}
$$

The bideterminant [20] or, equivalently, the determinant in the symmetrized semiring $\mathcal{S}^{2}[30,15]$ of a square matrix $A$ is the ordered pair

$$
\operatorname{det} A \stackrel{\text { def }}{=}\left(\operatorname{det}^{+} A, \operatorname{det}^{-} A\right) \text {. }
$$

We say that the determinant is balanced if $\operatorname{det}^{+} A=\operatorname{det}^{-} A$, otherwise it is unbalanced. Let $I, J \subseteq \mathbb{N}$ denote (possibly infinite) sets of row and column indices. Given an $I \times J$ matrix $A$ and two subsets $I^{\prime}=\left\{i_{1}, \ldots, i_{r}\right\} \subseteq I, J^{\prime}=\left\{j_{1}, \ldots, j_{s}\right\} \subseteq J$, with $i_{1}<\cdots<i_{r}, j_{1}<\cdots<j_{s}$, we denote by $A\left[I^{\prime} \mid J^{\prime}\right]$ or $A\left[i_{1}, \ldots, i_{r} \mid j_{1}, \ldots, j_{s}\right]$ the $r \times s$ submatrix $\left(A_{i_{m} j_{t}}\right)_{1 \leq m \leq r, 1 \leq t \leq s}$. Let $\|X\|$ denote the cardinality of a set $X$. The minor rank $\operatorname{rk}_{m} A$ of a matrix $A$ is the supremum of the order of the finite square submatrices of $A$ with unbalanced determinant

$$
\operatorname{rk}_{m} A=\sup \left\{r>0 ; \begin{array}{c}
\exists I^{\prime} \subseteq I, \exists J^{\prime} \subseteq J,\left\|I^{\prime}\right\|=\left\|J^{\prime}\right\|=r \\
\text { and } \operatorname{det}^{+} A\left[I^{\prime} \mid J^{\prime}\right] \neq \operatorname{det}^{-} A\left[I^{\prime} \mid J^{\prime}\right]
\end{array}\right\}
$$

\footnotetext{
${ }^{2}$ Row rank, column rank, Schein rank are distinct standard notions for Boolean matrices [24], which can also be defined in general semirings. Moreover, in the $\mathbb{R}_{\max }$ case, other rank notions have been used in relation to the uniqueness of solutions of linear systems [5], [15, Chapter 3, section 10].
} 
and $\operatorname{rk}_{m} A=0$ if no submatrix of $A$ with unbalanced determinant exists. The following result taken from $[15,16]$ is a semiring weak version of a well-known result over fields $[13,3]$.

THEOREM 3.1 (minor bound). The dimension of a linear realization of a sequence $h$ is not less than the minor rank of its Hankel matrix.

Hence,

$$
\operatorname{rk}_{m} \mathcal{H} \leq \operatorname{mlr}(h)
$$

This result holds in an arbitrary commutative semiring $\mathcal{S}$ (and not only in $\mathbb{R}_{\max }$ ). It is purely combinatorial in nature. We will prove it as a consequence of the following semiring version $[15,16]$ of the classical Binet-Cauchy identity [25, section 2.4.14].

LEMMA 3.2 (Binet-Cauchy formula). Let $\mathcal{S}$ be an arbitrary commutative semiring. Let $A \in \mathcal{S}^{n \times r}, B \in \mathcal{S}^{r \times p}$. For all subsets $I \subseteq\{1, \ldots, n\}, J \subseteq\{1, \ldots, p\}$ of $k$ elements, we have

$$
\begin{aligned}
& \operatorname{det}^{+}(A B)[I \mid J] \oplus \bigoplus_{K^{\prime}}\left(\operatorname{det}^{+} A\left[I \mid K^{\prime}\right] \operatorname{det}^{-} B\left[K^{\prime} \mid J\right] \oplus \operatorname{det}^{-} A\left[I \mid K^{\prime}\right] \operatorname{det}^{+} B\left[K^{\prime} \mid J\right]\right) \\
= & \operatorname{det}^{-}(A B)[I \mid J] \oplus \bigoplus_{K}\left(\operatorname{det}^{+} A[I \mid K] \operatorname{det}^{+} B[K \mid J] \oplus \operatorname{det}^{-} A[I \mid K] \operatorname{det}^{-} B[K \mid J]\right)
\end{aligned}
$$

where the sums are taken over all the $k$-element subsets $K, K^{\prime} \subseteq\{1, \ldots, r\}$. By convention, these two sums are equal to the zero element of $\mathcal{S}$ if $k>r$.

More generally, a folklore "transfer principle" [15, Chapter 1] asserts that usual ring identities admit semiring analogues whenever written without minus sign. Such semiring analogues can be obtained by direct combinatorial means (Zeilberger [33] proves the case $n=p=r$ of (3.2); the general case can be proved along the same lines). They can also be deduced formally from their classical ring versions, following an algebraic argument due to Reutenauer and Straubing [32], which we reproduce here for the sake of completeness. Note also that a different Binet-Cauchy identity in the $(\max ,+)$ semiring (valid for permanents) has been given by Bapat [2].

Proof of Lemma 3.2. Let $X=\left\{a_{i j}^{\prime}, b_{k l}^{\prime} ; 1 \leq i \leq n ; 1 \leq j, k \leq r ; 1 \leq l \leq p\right\}$ denote a family of distinct commuting indeterminates, and consider the semiring of (formal) commutative polynomials with coefficients in $\mathbb{N}$ and indeterminates $x \in X$ : $\mathcal{S}^{\prime}=\mathbb{N}[X] \subseteq \mathbb{Z}[X]$. Introduce the two matrices $A^{\prime}=\left(a_{i j}^{\prime}\right), B^{\prime}=\left(b_{k l}^{\prime}\right)$ with entries in $\mathcal{S}^{\prime}$. We first note that the identity (3.2) holds for $A^{\prime}$ and $B^{\prime}$. Indeed, using the invertibility of the addition of $\mathbb{Z}[X]$, the identity (3.2) for $A^{\prime}$ and $B^{\prime}$ is equivalent to the conventional Binet-Cauchy identity which is known to be valid in the ring $\mathbb{Z}[X]$ :

$$
\operatorname{det} A^{\prime} B^{\prime}[I \mid J]=\sum_{K} \operatorname{det} A^{\prime}[I \mid K] \operatorname{det} B^{\prime}[K \mid J] .
$$

Now, there is a unique morphism of semirings $\varphi: \mathcal{S}^{\prime} \rightarrow \mathcal{S}$, such that $\forall i, j, k, l, \varphi\left(a_{i j}^{\prime}\right)=$ $a_{i j}, \varphi\left(b_{k l}^{\prime}\right)=b_{k l}$. This morphism transforms the identity (3.2), applied to the matrices $A^{\prime}, B^{\prime}$ with entries in $\mathcal{S}^{\prime}$, to the required identity for the matrices $A, B$ with entries in $\mathcal{S}$.

Proof of Theorem 3.1. Consider a linear realization $(A, b, c)$ of $h$ of dimension $r$. Let $\mathcal{O}=\left[c, c A, c A^{2}, \ldots\right]^{T}$ and $\mathcal{C}=\left[b, A b, A^{2} b, \ldots\right]$, and consider two finite subsets $I, J \subseteq \mathbb{N}$. Applying the Binet-Cauchy identity to the finite size factorization $\mathcal{H}[I \mid J]=$ $\mathcal{O}[I \mid 1 \ldots r] \mathcal{C}[1 \ldots r \mid J]$ following from $\mathcal{H}=\mathcal{O C}$, we get $\operatorname{det}^{+}(\mathcal{H}[I \mid J])=\operatorname{det}^{-}(\mathcal{H}[I \mid J])$ if $|I|=|J|>r$. Hence, $\operatorname{rk}_{m} \mathcal{H} \leq r$. 
4. Preliminary majorization results. The proof of Theorem 2.1 will use standard convexity results that we recall here. The following famous result, due to Hardy, Littlewood, and Pólya, states the equivalence of two possible definitions of the relation of majorization.

THEOREM 4.1 (see [23, section 2.20], [26]). Let $\alpha_{1} \leq \alpha_{2} \leq \cdots \leq \alpha_{k}$ and $\alpha_{1}^{\prime} \leq$ $\alpha_{2}^{\prime} \leq \cdots \leq \alpha_{k}^{\prime}$ be real nonnegative numbers. The following two statements are equivalent:

1. $\alpha_{1}+\cdots+\alpha_{k}=\alpha_{1}^{\prime}+\cdots+\alpha_{k}^{\prime}$ and $\alpha_{\nu}+\cdots+\alpha_{k} \geq \alpha_{\nu}^{\prime}+\cdots+\alpha_{k}^{\prime}$ for all $\nu, 2 \leq \nu \leq k$.

2. There exists a doubly stochastic ${ }^{3}$ matrix $P$ such that $\alpha^{\prime}=P \alpha$ where $\alpha=\left(\alpha_{1}, \ldots, \alpha_{k}\right)^{T}, \alpha^{\prime}=\left(\alpha_{1}^{\prime}, \ldots, \alpha_{k}^{\prime}\right)^{T}$.

We write $\alpha^{\prime} \prec \alpha$ and say that $\alpha^{\prime}$ is majorized by $\alpha$ when these two equivalent statements hold.

The following majorization inequality is standard $[23,26]$. We single out the strict-inequality case for further use.

THEOREM 4.2. Let $g$ be a convex function $\mathbb{R} \rightarrow \mathbb{R}$.

1. If $\alpha^{\prime} \prec \alpha$, then

$$
\sum_{i} g\left(\alpha_{i}^{\prime}\right) \leq \sum_{i} g\left(\alpha_{i}\right)
$$

2. Take $P=\left(P_{j m}\right)$ such that $\alpha^{\prime}=P \alpha$ as in Theorem 4.1. If for some $j$ the restriction of $g$ to the set $\left\{\alpha_{m} \mid P_{j m} \neq 0\right\}$ does not coincide with an affine function, then the strict inequality holds in (4.1).

Proof. Classically, (4.1) is obtained by summing up the convexity inequalities

$$
\forall j, g\left(\alpha_{j}^{\prime}\right) \leq \sum_{m} P_{j m} g\left(\alpha_{m}\right) .
$$

The strict inequality in (4.1) follows from the strict inequality in (4.2), as soon as $j$ satisfies condition 4.2 of the theorem.

5. Proof of Theorem 2.1. Suppose that the sequence $h_{0}, h_{1}, \ldots$ is convex. To prove Theorem 2.1, it remains to show that $\operatorname{mlr}(h) \geq \operatorname{mpr}(h)$. We will assume that $\mathrm{m} \operatorname{lr}(h)<\infty$ (otherwise, there is nothing to prove). Then, the existence of a polynomial realization follows readily from the convexity of $h$ together with (2.1). Let $p$ be such a polynomial realization satisfying

$$
\forall x \in \mathbb{R}, \quad p(x)=\max _{1 \leq i \leq r} \ell_{i}(x)=\max _{1 \leq i \leq r}\left(\alpha_{i}+\beta_{i} x\right),
$$

with

$$
\beta_{1}<\beta_{2}<\cdots<\beta_{r} \quad \text { and } \quad r=\operatorname{mpr}(h)
$$

We set

$$
\begin{aligned}
& I_{0}=\left[x_{0}, z_{0}\right] \stackrel{\text { def }}{=}\left\{x \in \mathbb{N} \mid p(x)=\ell_{1}(x)\right\} \quad \text { and } \\
& I_{i}=\left[x_{i}, z_{i}\right] \stackrel{\text { def }}{=}\left\{x \in \mathbb{N} \mid p(x)=\ell_{i+1}(x)>\ell_{i}(x)\right\} \quad \text { for } i=1, \ldots, r-1 \text {, }
\end{aligned}
$$

\footnotetext{
3 "Doubly stochastic" refers, as usual, to a matrix with nonnegative entries in which both the row and column sums are equal to 1 .
} 
with $x_{i}, z_{i} \in \mathbb{N}$, except the last value $z_{r-1}=\infty$. (The fact that $I_{i}$ is nonempty follows from the minimality of $r$. The fact that it is an interval is immediate due to the convexity of $\max _{i} \ell_{i}$.) Note that $\alpha_{1}>\alpha_{i}$ for $i=2, \ldots, r$ (otherwise, using (5.2), we get $\ell_{1}(k) \leq \ell_{i}(k) \forall k \geq 0$, which contradicts the minimality or $r$ ). Hence, $p(0)=\alpha_{1}=\ell_{1}(0)$, and thus $x_{0}=0$. To summarize,

$$
0=x_{0} \leq z_{0}<x_{1} \leq z_{1} \cdots \leq z_{r-2}<x_{r-1} .
$$

The following elementary lemma states the existence of a minimal polynomial realization in which each line passes through at least two consecutive points.

LEMMA 5.1. There exists a minimal polynomial realization (5.1) such that

$$
x_{i+1} \geq x_{i}+2 \quad \text { for } i=0, \ldots, r-2 .
$$

The proof of the lemmas is at the end of this part.

COROLlARY 5.2. If $p$ is the polynomial realization in Lemma 5.1, then $p$ does not coincide with an affine function on $\left[x_{i}, x_{i+1}\right]$ for $i=0, \ldots, r-2$.

Without loss of generality, we suppose that the polynomial realization $p$ satisfies the conditions of Lemma 5.1 and Corollary 5.2. We set

$$
u_{i} \stackrel{\text { def }}{=} x_{i}-i \quad \text { for } i=0, \ldots, r-1 .
$$

We get from (5.5) that

$$
u_{0}<u_{1}<\cdots<u_{r-1} .
$$

LEMMA 5.3. Let $M=\mathcal{H}\left[0,1, \ldots, r-1 \mid u_{0}, u_{1}, \ldots, u_{r-1}\right]$. We have

$$
\operatorname{det}^{+} M=\bigotimes_{i=0}^{r-1} p\left(x_{i}\right)>\operatorname{det}^{-} M \text {. }
$$

Thus, $\mathcal{H}$ contains an $r \times r$ submatrix with unbalanced minor, i.e., $\operatorname{rk}_{m} \mathcal{H} \geq r$, which together with the minor bound gives $\operatorname{mlr}(h) \geq \operatorname{rk}_{m} \mathcal{H} \geq r=\operatorname{mpr}(h)$, and Theorem 2.1 follows.

Proof of Lemma 5.1. We show that there exists a minimal polynomial realization of the form (5.1) with

$$
z_{i} \geq x_{i}+1 \quad \text { for } i=0, \ldots, r-1
$$

We start from an arbitrary minimal realization (5.1). Let $i_{0}=\min \left\{i \mid x_{i}=z_{i}\right\}$. By replacing $\ell_{i_{0}+1}$ with the affine map $\ell$ passing through the two points $\left(x_{i_{0}}, h_{x_{i_{0}}}\right),\left(x_{i_{0}}+\right.$ $\left.1, h_{x_{i_{0}}+1}\right)$, we obtain a new decomposition of the form (5.1) with $i_{0}<i_{0}^{\prime}=\min \{j \mid$ $\left.x_{j}^{\prime}=z_{j}^{\prime}\right\}$, where $x_{j}^{\prime}, z_{j}^{\prime}$ are points defined by (5.3) for the new polynomial realization. Indeed, $x_{i}^{\prime}=x_{i}, z_{i}^{\prime}=z_{i}$ for all $i<i_{0}$ and $x_{i_{0}}^{\prime}=x_{i_{0}}, z_{i_{0}}^{\prime} \geq x_{i_{0}}+1>x_{i_{0}}=x_{i_{0}}^{\prime}$. Note that $\ell \neq \ell_{i_{0}+2}$; otherwise $\ell_{i_{0}+1}$ could be removed from $p$ and the arising function would still be a polynomial realization of $h$ which contradicts the minimality of $p$. After a finite number of such replacements, we get $z_{i}>x_{i}$ for all $i$, so that (5.5) becomes satisfied.

Proof of Lemma 5.3. From the definition of $M$ we have

$$
M=\left(h_{i+u_{j}}\right)=\left(p\left(i+u_{j}\right)\right)_{i, j=0, \ldots, r-1} .
$$


We prove that for all permutations $\sigma$ of $0, \ldots, r-1$ such that $\sigma \neq \mathrm{Id}$,

$$
\bigotimes_{i=0}^{r-1} p\left(i+u_{i}\right)>\bigotimes_{i=0}^{r-1} p\left(i+u_{\sigma(i)}\right)
$$

Clearly, it is sufficient to show that for all elementary cycles $c=\left(i_{1}, \ldots, i_{k}\right)(k \geq 2)$,

$$
\begin{aligned}
& p\left(i_{1}+u_{i_{1}}\right) \otimes p\left(i_{2}+u_{i_{2}}\right) \otimes \cdots \otimes p\left(i_{k}+u_{i_{k}}\right) \\
& >p\left(i_{1}+u_{i_{2}}\right) \otimes p\left(i_{2}+u_{i_{3}}\right) \otimes \cdots \otimes p\left(i_{k}+u_{i_{1}}\right)
\end{aligned}
$$

or with the conventional notation

$$
\begin{aligned}
& p\left(i_{1}+u_{i_{1}}\right)+p\left(i_{2}+u_{i_{2}}\right)+\cdots+p\left(i_{k}+u_{i_{k}}\right) \\
& >p\left(i_{1}+u_{i_{2}}\right)+p\left(i_{2}+u_{i_{3}}\right)+\cdots+p\left(i_{k}+u_{i_{1}}\right) .
\end{aligned}
$$

Let $\alpha_{1}, \ldots, \alpha_{k}$, with $\alpha_{1}<\cdots<\alpha_{k}$, denote the sequence obtained by reordering the $x_{l}=i_{l}+u_{i_{l}}$ (since $x_{t}<x_{s} \Leftrightarrow t<s \Leftrightarrow u_{t}<u_{s}, i_{l}$ and $u_{i_{l}}$ are ordered in the same way), and let $\alpha_{1}^{\prime}, \ldots, \alpha_{k}^{\prime}$ with $\alpha_{1}^{\prime} \leq \cdots \leq \alpha_{k}^{\prime}$ denote the sequence obtained by reordering the $i_{l}+u_{i_{l+1}}$.

We claim that $\alpha^{\prime} \prec \alpha$. Indeed, condition 1 of Theorem 4.1 is satisfied, because $\alpha_{\nu}+\cdots+\alpha_{k}$ is equal to the sum of the $k-\nu+1$ highest possible values of $i_{l}$ and $u_{i_{l}}$; hence, it is greater than $\alpha_{\nu}^{\prime}+\cdots+\alpha_{k}^{\prime}$ which is also the sum of $k-\nu+1$ values of $i_{l}$ and $u_{i_{l}}$.

Moreover, take $P$ such that $\alpha^{\prime}=P \alpha$ as in Theorem 4.1. Since $P$ is doubly stochastic, there is at least one $j \in\{1,2, \ldots, k\}$ such that $P_{j k} \neq 0$. Since $\alpha_{j}^{\prime} \leq$ $\alpha_{k}^{\prime}<\alpha_{k}$, we have $P_{j k} \neq 1$; thus there is at least one $m \in\{1,2, \ldots, k-1\}$ such that $P_{j m} \neq 0$. It remains to apply Corollary 5.2 together with the strict inequality case in Theorem 4.2 to get (5.11).

Example 1. The function $p(x)=\max (0,-3+x,-8+2 x,-22+4 x)$ is a polynomial realization of the sequence $h=0,0,0,0,1,2,4,6,10,14, \ldots$. From (5.3) we have

$$
\begin{aligned}
x_{0}=0, \quad x_{1}=4, & x_{2}=6, \quad x_{3}=8, \\
u_{0}=0, \quad u_{1}=3, & u_{2}=4, \quad u_{3}=5, \\
\operatorname{det} \mathcal{H}[0,1,2,3 \mid 0,3,4,5]=\operatorname{det} & {\left[\begin{array}{cccc}
0 & 0 & 1 & 2 \\
0 & 1 & 2 & 4 \\
0 & 2 & 4 & 6 \\
0 & 4 & 6 & 10
\end{array}\right]=(15,14), }
\end{aligned}
$$

which is unbalanced. Hence, $\operatorname{mir}(h)=4$ and a minimal linear realization of $h$ is $(A, b, c)$, where $A=\operatorname{diag}(0,1,2,4), c=(0,-3,-8,-22), b=(0,0,0,0)^{T}$. Note that this minimal realization is not unique.

6. Appendix. Now we develop a method for finding a polynomial realization of the minimal dimension.

Suppose that $h_{0}, h_{1}, \ldots$ is a convex sequence satisfying (2.1). Consider the points $P_{j}=\left[j, h_{j}\right], j=0,1, \ldots, M$, in the plane with Cartesian coordinate system. If $M=N+1$, then $p$ is a polynomial realization of $h$ iff $p(j)=h_{j}$ for $j=0, \ldots, M$, 
so that we may restrict our investigation to a method for finding a polynomial realization of the finite sequence $P_{0}, \ldots, P_{M}$. It is evident that to every polynomial realization (which in general may contain redundant monomials) we can assign another polynomial realization of no greater dimension in which every line (monomial) passes through at least two points of the set $\left\{P_{0}, \ldots, P_{M}\right\}$. Indeed, in what follows we consider only polynomial realizations which possess this property.

A subset $T$ of the set

$$
S=\left\{\left[j, h_{j}\right] \mid j=0,1, \ldots, M\right\}
$$

is called aligned if $\|T\| \geq 3$ and there exists a line $q$ such that

1. $T \subseteq q$,

2. $(S-T) \cap q=\emptyset$.

Note that an aligned subset of $S$ may not exist and that two different aligned subsets are either disjoint or have exactly one common point.

Let $T$ be a fixed aligned set of points lying on a line $q$. Since $\|T\| \geq 3,[j-$ $\left.1, h_{j-1}\right],\left[j, h_{j}\right],\left[j+1, h_{j+1}\right] \in q$ for some $j$.

Let $s(t)$ represent a line of an arbitrary polynomial realization which passes through $\left[j, h_{j}\right]$. Since

$$
h_{j-1} \geq s(j-1), \quad h_{j+1} \geq s(j+1),
$$

and $\left[j-1, h_{j-1}\right],\left[j, h_{j}\right],\left[j+1, h_{j+1}\right]$ are collinear, we have that $q$ coincides with $s$. Hence every polynomial realization contains each line passing through all points of an aligned subset, and thus in the construction of the minimal polynomial realization we must always include these lines. This concerns also the two special lines: one covering $\left[0, h_{0}\right],\left[1, h_{1}\right]$ and the other passing through $\left[M-1, h_{M-1}\right],\left[M, h_{M}\right]$, which by trivial reasons must be involved too.

The point $\left[j, h_{j}\right](0<j<M)$ is called a breaking point if $\left[j-1, h_{j-1}\right],\left[j, h_{j}\right],[j+$ $\left.1, h_{j+1}\right]$ are not collinear. Clearly, the first and last points of every aligned set are breaking points (except $\left[0, h_{0}\right],\left[M, h_{M}\right]$ ).

Consider a fixed set

$$
B=\left\{\left[r, h_{r}\right],\left[r+1, h_{r+1}\right], \ldots,\left[s, h_{s}\right]\right\}
$$

of consecutive breaking points which is maximal; i.e., both $\left[r, h_{r}\right]$ belongs to an aligned set or $r=1$ and $\left[s, h_{s}\right]$ belongs to an aligned set or $s=M-1$. Hence both $\left[r, h_{r}\right]$ and $\left[s, h_{s}\right]$ can be assumed to belong already to a line of the realization. Clearly, a line cannot pass through more than two consecutive breaking points. If $B$ consists of $k$ points, then the minimal number of lines joining pairs of consecutive points which contain all the points in $B$ (except for the extreme points $\left[r, h_{r}\right],\left[s, h_{s}\right]$ ) is

$$
\left\lceil\frac{k}{2}\right\rceil-1 \text {. }
$$

A self-evident strategy to achieve this lower bound is to take alternatively every other line consecutively joining the pairs of adjacent points starting by the line passing through

$$
\left[r+1, h_{r+1}\right], \quad\left[r+2, h_{r+2}\right] .
$$

The foregoing discussion justifies the following algorithm, which starts from $\left[0, h_{0}\right]$. (Note that $\ell \equiv(P, Q)$ reads, "line $\ell$ determined by the points $P$ and $Q . "$ ) 
Algorithm. MINIMAL POLYNOMIAL REALIZATION (MPR)

Input: Finite sequence $h_{0}, \ldots, h_{M}$ of real numbers, with $M \geq 3$.

Output: Polynomial realization of $h$ of minimal dimension represented by the lines $\ell_{1}, \ell_{2}, \ldots ;$ "no" if $h$ is not convex.

(1) $r:=1, s:=0, j:=0, P_{i}=\left[i, h_{i}\right],(i=0,1, \ldots, M)$.

(2) Accept $\ell_{r} \equiv\left(P_{j}, P_{j+1}\right)$.

(3) $r:=r+1$.

(4) Let $j(j>s)$ be the least index for which $\ell_{r-1}$ does not pass through $P_{j}$ (if no such exists then stop).

(5) If $P_{j}$ is below $\ell_{r-1}$ then stop ("no").

(6) If $j=M$ then go to (8).

(7) $s:=j$ and go to $(2)$.

(8) Accept $\ell_{r} \equiv\left(P_{j-1}, P_{j}\right)$, stop.

Example 2. Input: $h=1,0,-1,-1,0,2,5,8 .(M=7)$

$P_{0}=(0,1), P_{1}=(1,0), P_{2}=(2,-1), P_{3}=(3,-1), P_{4}=(4,0), P_{5}=(5,2)$, $P_{6}=(6,5), P_{7}=(7,8)$.

The algorithm MPR produces the following:

$r=1, s=0$

accept $\ell_{1} \equiv\left(P_{0}, P_{1}\right) \quad$ accept $\ell_{2} \equiv\left(P_{3}, P_{4}\right) \quad$ accept $\ell_{3} \equiv\left(P_{5}, P_{6}\right)$

$r=2 \quad r=3 \quad r=4$

$j=3 \quad j=5$

$s=3 \quad s=5$

$p_{1}(t)=-t+1\left(\right.$ line $\left.\ell_{1}\right)$

stop

$p_{2}(t)=t-4\left(\right.$ line $\left.\ell_{2}\right)$

$p_{3}(t)=3 t-13\left(\right.$ line $\left.\ell_{3}\right)$

$A=\operatorname{diag}(-1,1,3), c=(1,-4,-13), b=(0,0,0)^{T}$.

\section{REFERENCES}

[1] F. Baccelli, G. Cohen, G. Olsder, And J. QuAdrat, Synchronization and Linearity, John Wiley, New York, 1992.

[2] R. B. BAPAT, Permanents, max algebra and optimal assignment, Linear Algebra Appl., 226/228 (1995), pp. 73-86.

[3] J. Berstel and C. Reutenauer, Rational Series and Their Languages, Springer-Verlag, New York, 1988.

[4] G. Birkhoff and S. Maclane, A Survey of Modern Algebra, Macmillan, New York, 1965.

[5] R. Cuninghame-Green, Minimax Algebra, Lecture Notes in Econom. and Math. Systems 166, Springer-Verlag, New York, 1979.

[6] R. Cuninghame-Green, Algebraic realization of discrete dynamic systems, in Proc. of the 1991 IFAC Workshop on Discrete Event System Theory and Applications in Manufacturing and Social Phenomena, Shenyang, China, June 1991.

[7] R. Cuninghame-Green and P. Butkovič, Discrete-event dynamic systems: The strictly convex case, Ann. Oper. Res., 57 (1995), pp. 45-63.

[8] R. Cuninghame-Green And P. MeiJer, An algebra for piecewise-linear minimax problems, Discrete Appl. Math., 2 (1980), pp. 267-294.

[9] B. De Schutter, Max-Algebraic System Theory for Discrete-Event Systems, Ph.D. thesis, Katholieke Univ. Leuven, Feb. 1996.

[10] B. De Schutter And B. De Moor, The characteristic equation and minimal state space realization of siso systems in the max algebra, in Proc. of the 11th Conf. on Anal. and Opt. of Systems: Discrete-Event Systems, Lect. Notes. in Control and Inform. Sci. 199, Springer-Verlag, New York, 1994.

[11] B. De Schutter And B. De Moor, Minimal realization in the max algebra is an extended linear complementarity problem, Sys. Control Lett., 25 (1995), pp. 103-111. 
[12] B. De Schutter And B. De Moor, Minimal state space realization of mimo systems in the max algebra, in Proc. of the 3rd European Control Conference, Roma, Italy, Sept. 1995, pp. 411-416.

[13] M. Fliess, Matrices de Hankel, J. Math. Pures. Appl., 53 (1974), pp. 197-222.

[14] M. Fliess, Séries rationnelles positives et processus stochastiques, Ann. Inst. H. Poincaré, XI (1975), pp. 1-21.

[15] S. Gaubert, Théorie des systèmes linéaires dans les diö̈des, thèse, École des Mines de Paris, 1992.

[16] S. Gaubert, On Rational Series in One Variable over Certain Dioids, Rapport de Recherche 2162, INRIA, Le Chesnay, France, Jan. 1994.

[17] S. Gaubert, Rational series over dioids and discrete-event systems, in Proc. of the 11th Conf. on Anal. and Opt. of Systems: Discrete-Event Systems, Lect. Notes. in Control and Inform. Sci. 199, Springer-Verlag, New York, 1994.

[18] J. S. Golan, The Theory of Semirings with Applications in Mathematics and Theoretical Computer Science, Pitman Monogr. Surveys Pure Appl. Math. 54, Longman Scientific and Technical Publishers, Harlow, U.K., 1992.

[19] M. Gondran And M. Minoux, Graphes et algorithmes, Eyrolles, Paris, 1979. Engl. transl. Graphs and Algorithms, John Wiley, New York, 1984.

[20] M. Gondran And M. Minoux, Linear algebra in dioids: A survey of recent results, Ann. Discrete Math., 19 (1984), pp. 147-164.

[21] J. Gunawardena, ED., Idempotency, Publications of the Newton Institute, Cambridge University Press, London, 1996.

[22] G. Hansel And D. Perrin, Mesures de probabilités rationnelles, in Mots, M. Lothaire, ed., Hermes, Paris, 1990.

[23] G. Hardy, J. Littlewood, And G. Pólya, Inequalities, Cambridge University Press, London, 1934, reprinted 1973.

[24] K. KIM, Boolean Matrix Theory and Applications, Marcel Dekker, New York, 1982.

[25] M. Marcus And H. Minc, A Survey of Matrix Theory and Matrix Inequalities, Allyn and Bacon, Boston, 1964.

[26] A. Marshall And I. Olkin, Inequalities: Theory of Majorization and Its Applications, Academic Press, New York, 1979.

[27] V. Maslov and S. Samborskil̆, Eds., Idempotent analysis, Adv. in Soviet Math. 13, AMS, Providence, RI, 1992.

[28] G. OlSDER, On the characteristic equation and minimal realizations for discrete-event dynamic systems, in Analysis and Optimization of Systems, A. Bensoussan and J. Lions, eds., in Lecture Notes in Control and Inform. Sci. 83, Springer-Verlag, New York, 1986, pp. 189201.

[29] G. OLSDER, Some results on the minimal realization of discrete-event systems, in 25th IEEE Conf. on Decision and Control, Athens, Greece, 1986.

[30] M. Plus, Linear systems in (max, +)-algebra, in Proceedings of the 29th Conference on Decision and Control, Honolulu, Dec. 1990.

[31] X. QI And W. Chen, The minimal realization of discrete-event systems, in Proc. of the 1991 IFAC Workshop on Discrete Event System Theory and Applications in Manufacturing and Social Phenomena, Shenyang, China, June 1991, pp. 29-33.

[32] C. Reutenauer And H. Straubing, Inversion of matrices over a commutative semiring, J. Algebra, 88 (1984), pp. 350-360.

[33] D. Zeilberger, A combinatorial approach to matrix algebra, Discrete Math., 56 (1985), pp. $61-72$. 